\title{
Evaluation of TPMS Signal Propagation in a Heavy Commercial Vehicle Environement
}

\author{
Ahmad Rida Tawakuli \\ Secan-Lab \\ University of Luxembourg \\ Luxembourg \\ ahmad.tawakuli@uni.lu
}

\author{
Ridha Soua \\ IEEE member \\ Luxembourg \\ ridha.soua@gmail.com
}

\author{
Thomas Engel \\ Secan-Lab \\ University of Luxembourg \\ Luxembourg \\ thomas.engel@uni.lu
}

\begin{abstract}
This paper aims to evaluate the performance of the state-of-the-art Tire Pressure Monitoring System (TPMS) in a heavy commercial vehicle environment. We adopt a novel qualitative approach to determine the radio frequency (RF) signal propagation characteristics of high frequency antennas used in a state of the art TPMS solution [1]. We outline the effect of both the tire and wheel on the RF signal and system function, and compare it to the propagation in a free space. The paper quantifies the antenna $R F$ propagation using finite element method (FEM) and using realistic models of the sensor, the wheel and the commercial tire. The adverse effect of the heavy vehicle environment on the signal propagation is described. Finally, the paper questions the feasibility of conventional TPMS in commercial vehicle, and calls for novel communication technologies to enable future use case such as smart tires.
\end{abstract}

Keywords - TPMS, FEM, heavy commercial vehicle, truck, RF, propagation, antennas, automotive, tires, sensors.

\section{INTRODUCTION}

Since its introduction more than 20 years ago, Tire Pressure Monitoring System (TPMS) has been a crucial safety feature in passenger cars and has now become a mandatory feature of all new registered cars. On the other hand, its application in heavy commercial vehicles has been limited to short lived solutions such as the case with the Yokohama Airwatch [2] or limited to tires fitted on the main lorry/tractor axles such as the case of continental ContipressureCheck [3]. These two solutions do not provide an efficient solution for the Tractor-Trailer heavy commercial vehicles, the most common type of vehicles used by logistics fleets. EU regulations mandate that all commercial vehicles be equipped by TPMS by 2022 and all Tractor-Trailer vehicles by 2024 [4]. Therefore, efforts have been stepped up in the past few years to develop reliable TPMS (reliable signal propagation) in this harsh environment.

Figure 1 presents the layout of a commercial heavy vehicle TPMS system. Most of the state of the art solutions propose Radio frequency communication (RF) between a transmitter in the tire pressure sensor (1) that is mounted on each tire, and a receiver units (2)(4) mounted on the tractor and trailer frames of commercial vehicles. Tire pressure information is transmitted by each sensor to the receiver units, which in turn relays the data to the interface unit where the data is interpreted and indicated to the driver (3) in the form of audio and visual indications [1][2][3]. While it is well known to RF engineers that dense metal environments and large surfaces cause severe attenuations to the RF signals by absorbing losses as well as refraction and multipath effect [5], the extent of this attenuation has not been quantified yet on heavy commercial vehicles.

To provide a realistic and factual evaluation of a TPMS solution provided by Goodyear [1], the RF propagation of the transmitter unit in the application environment has to be calculated as accurately as possible. This is a very complex process which involves solving the Maxwell electromagnetic wave equations for the complex multilayer and multi material structure of the tire, all of which have different electrical properties [5]. Previous Finite element analysis of commercial vehicle TPMS were based on academic approximations [6], [7] or simplified models [8], [9], rather than actual industrial data and precise models. This has led to feasible results on a theoretical level but cannot be demonstrated with a functioning system. In this paper, we model the transmitter used in Goodyear TPMS solution and use a highly detailed model of an actual heavy commercial vehicle tire and wheel. Our aim is to carry out the most accurate evaluation of signal propagation in a heavy vehicle environment. The remainder of this paper is organized as follow: Section II evaluates RF propagation in a free-space, and will be considered as a baseline measurement. Section III evaluates RF propagation inside the wheel and the tire of a truck. Next, we analyze obtained results taking into account other factors that affect signal propagation such as weather and vehicle structure. Section V concludes the paper.

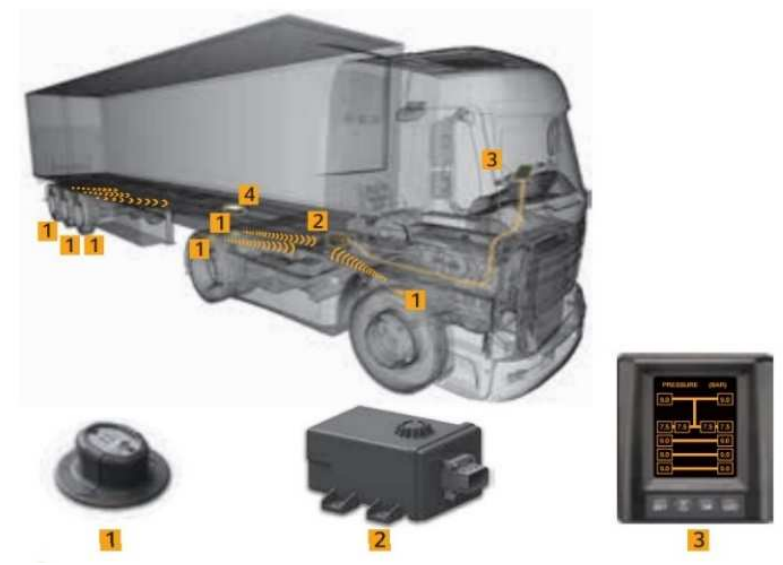

Figure 1: Layout of a Commercial vehicle TPMS System [3]

\section{EVALUATION OF RF PROPAGATION IN FREE-SPACE}

\section{A. Modeling of the TPMS Transmitter unit}

To have a benchmark for comparing RF propagation in the truck tire and wheel combination, we first created a detailed model of the transmitter used by a state-of-the-art TPMS sensor [1]. We use the Ansys HFSS (high-frequency structure simulator) electromagnetics suite [11] to design the transmitter and analyze the signal propagation. The transmitter comprises of an integrated circuit with a temperature sensor, a pressure sensor and an electrically small transmission antenna. The transmitter operates on the European TPMS standard frequency range of $433 \mathrm{MHz}$. The sensor is encased by a hard plastic mold $3-4 \mathrm{~mm}$ thick, and attached to the wheel circumference with a braided steel strap. This transmitter is the most advanced in the commercial 
vehicle TPMS sensors. Other sensor models are attached to the tire carcass using a special adhesive.

We used some aspects of the antenna design proposed by authors of [12]. To model the transmitter unit. The antenna is of aluminum, built into a PCB board and shorted to ground. The wavelength $(\lambda)$ at the operating frequency is $69 \mathrm{~cm}$, the typical antenna size for efficient radiation at this wavelength is $34.56 \mathrm{~cm}$ at $\frac{\lambda}{2}$ or $17.28 \mathrm{~cm}$ at $\frac{\lambda}{4}$. We use a transmitter antenna with an overall length equal to $4 \mathrm{~cm}$, which is considerably smaller than the typical antenna sizes. Our antenna presents the characteristics of electrical small antennas, and thus it offers low antenna gain compared to an isotropic antenna of sufficient size. It is important to note that such antennas use the attached surface as an extension of the radiating surface, increasing the antenna gain. This benefit is not available in free space analysis. On the other hand, the TPMS sensor size of around 4-6 cm prevents the use of larger antennas, and TPMS manufacturers found this design to be adequate through trial and error tests and RSSI measurements [12]. Actual analysis in our considered environment has not been carried out. We complete a detailed model of the transmitter as shown in figure 2 using information and measurements of the actual transmitter.

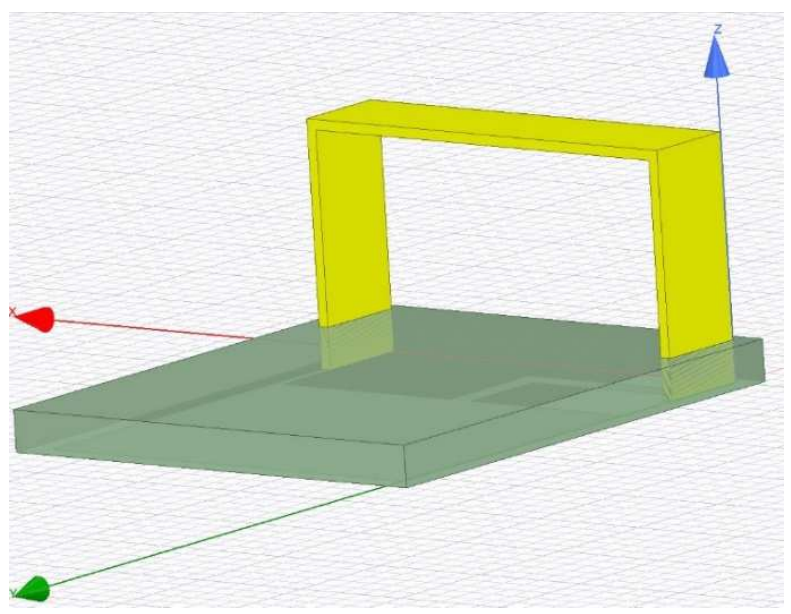

Figure 2: Transmitter unit design used in the FEM Analysis

\section{B. Analysis of RF propagation in free space}

Our analysis uses the actual transmitter design parameters rather than theoretical design parameters. These parameters may not produce optimal characteristics in free space neither optimal gain values, but are the most suitable during the manufacturer tests. The incident power takes into consideration the interference regulations and the sensor battery life. The matching network was calculated using the method describe in the technical report [13]. Parameters are highlighted in Table 1.

The Antenna shows the characteristics of an electrically small antenna [5]. The Antenna gain is negative due the antenna type, ranging from $-16.7 \mathrm{~dB}$ to $-25.8 \mathrm{~dB}$. As depicted in Figure 3, the propagation pattern is omni-directional with null points on antenna port and ground point. The $\mathrm{H}$ field is greatly reduced in this design due to the ground being in close proximity. The E field is significantly stronger than the $\mathrm{H}$ field. Both fields are negligible at this operating frequency and power, radiating only millimeters around the antenna. The previous comparison between the whip antenna design and the single loop shorted to ground showed higher gain with the whip antenna [12]. But the choice of this design by the manufacturer might also be due to a better propagation in the metal environment, as well as better suitability for encasing in molds as it is a more rigid design. The propagation pattern in free spaces is illustrated in Figure 3 and Figure 4, which present a better visualization of the propagation relative to the wheel and tire models thought both models are not included in the analysis.

Table 1: Antenna and Analysis parameters

\begin{tabular}{|l|l|}
\hline \multicolumn{1}{|c|}{ Parameter } & \multicolumn{1}{c|}{ Value } \\
\hline Incident Power & $21 \mathrm{~mW}$ via wave port \\
\hline Operating Frequency & $\begin{array}{l}\text { Matching network with } \\
\text { varying capacitance value } \\
\text { in the Pico farad range. }\end{array}$ \\
\hline Port impedance & $\begin{array}{l}\text { Electrically small } \\
\text { Monopole shorted to PCB } \\
\text { ground }\end{array}$ \\
\hline Antenna type & Aluminum \\
\hline Antenna material & FR4 \\
\hline PCB Material & 20 \\
\hline Minimum number of passes & $\begin{array}{l}350 \mathrm{MHz} \text { to 550MHz in 1 } \\
\text { MHz steps. Discrete Sweep }\end{array}$ \\
\hline Sweep setting &
\end{tabular}

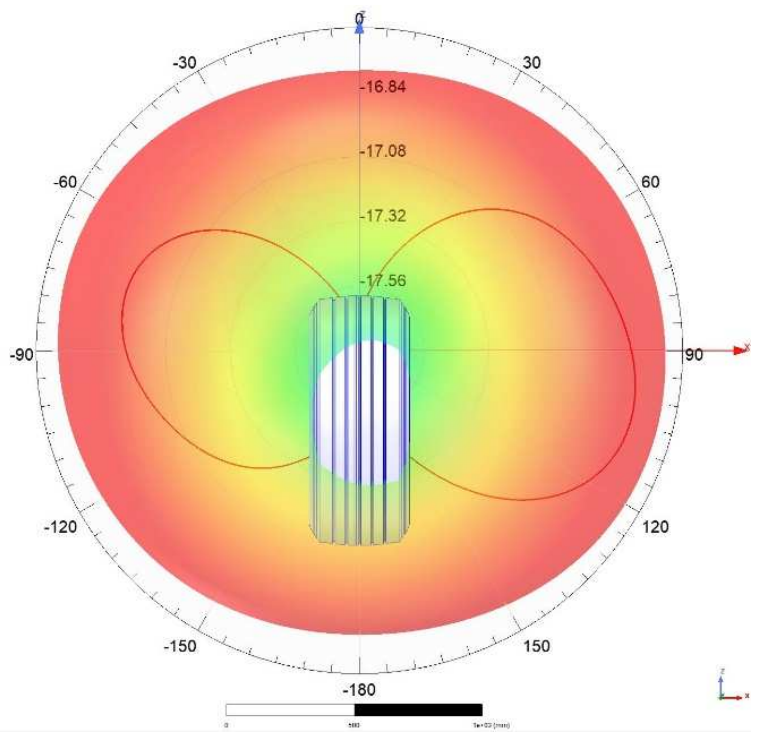

Figure 3: Propagation in free space along the $X$-axis relative to the tire model 


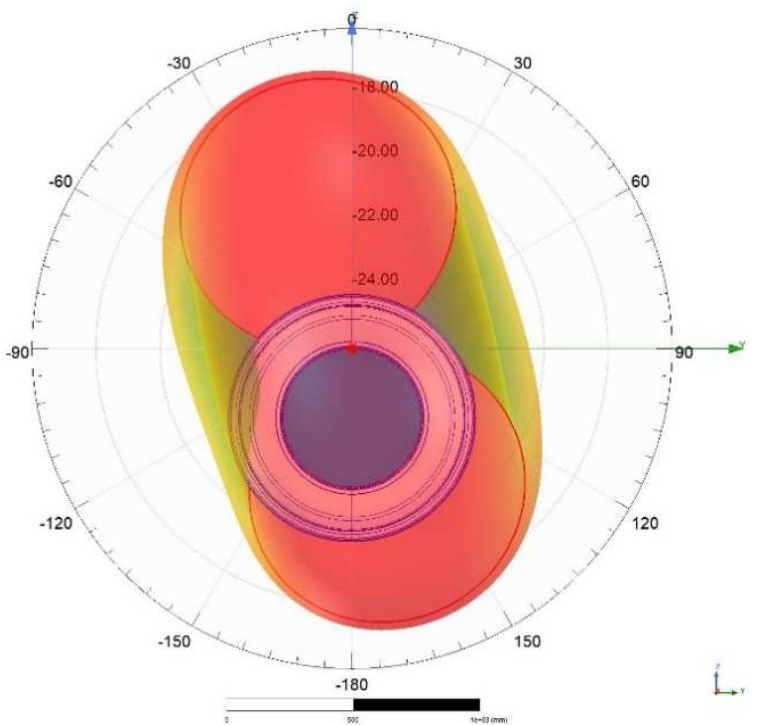

Figure 4: Propagation in free space along the Y-axis relative to the tire model

\section{EVALUATION OF RF PROPAGATION IN A HEAVY VEHICLE ENVIRONMENT}

\section{A. Modelling a commercial vehicle tire and wheel}

Before starting the complex FEM analysis of RF propagation inside the wheel and tire, we have to determine the main cause of signal attenuation. Previous FEM analysis represented the tire as a single structure comprising of a single material (i.e. Rubber) [6], [7], [10]. In fact, tires are a combination of different materials with a multi-layer structure as depicted in Figure 5.

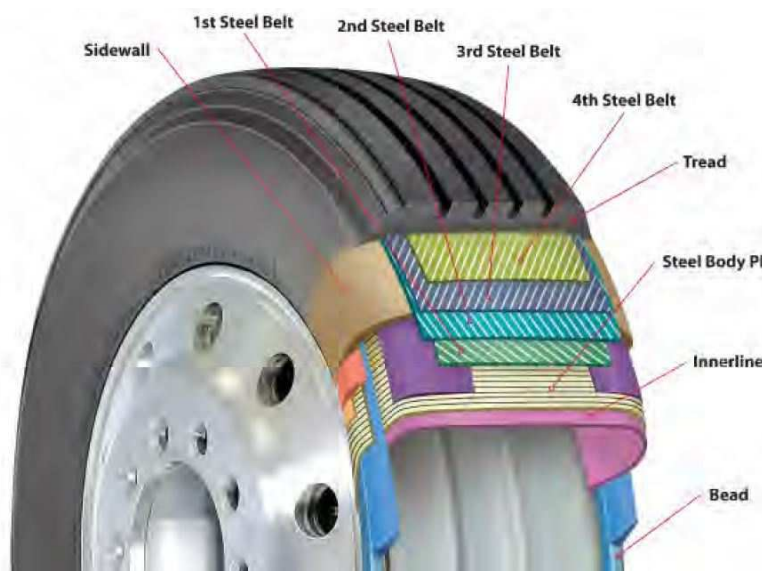

Figure 5: Internal structure of a commercial vehicle tire [14]

Most passenger car tires consist of several layers of carbon and silica enriched rubber compounds, with several layer of carbon steel mesh molded into the tire carcass to reinforce the structure, and separate from the rubber compound with layer of rayon or polyester [8][14][15]. Heavy vehicle tires present much greater challenges, as the carrying load requirements are much greater than passenger cars, hence the need for greater structural reinforcement of the tire. Heavy vehicle tires have more layers of steel reinforcement (effective thickness of 0.3$0.4 \mathrm{~mm}$ in passenger cars vs $0.4-0.9 \mathrm{~mm}$ in commercial tires) and not only in the tire tread area, but also in the shoulders and bead area. For testing, we use a tire which is being tested is an actual production model for wheels size 22.5 inch, used for long haul tractor-trailers. The tire consists of 54 different components and layers with some components overlapping. This tire has 4 layers of steel mesh. We determine the material used in each section of the tire and feed into the simulation, together with the tire specifications. In addition, the wheels used in passenger cars differ greatly from those used in heavy commercial vehicles. While passenger cars use aluminum wheels with a relatively low thickness of less than $5 \mathrm{~mm}$ and open spokes design, heavy commercial vehicles use cast carbon steel wheels of a closed design and higher thickness of 8 to $12 \mathrm{~mm}$ to support high loads. The wheels are either closed faced or hollow in the center to accommodate the protruding wheel hub, which represents a challenge for RF propagation.

We modify a tire model to reflect the various factors mentioned previously. We integrate the steel wheel with 9 inch width and 22.5 inch diameter into the model. Steel wheels of these dimensions are commonly used in European tractortrailer models. The wheel flange edge sits flush with the tire chafer, while in actual fitting the side walls of the tire are protruding beyond the wheel while the tire chafers are pushed under the wheel flange to create the air tight seal. This cannot be modelled as it depends on tire pressure and side wall flexing in relation to the tread contact point with the ground and several other factors The detailed wheel and tire combination model is shown in Figure 6.

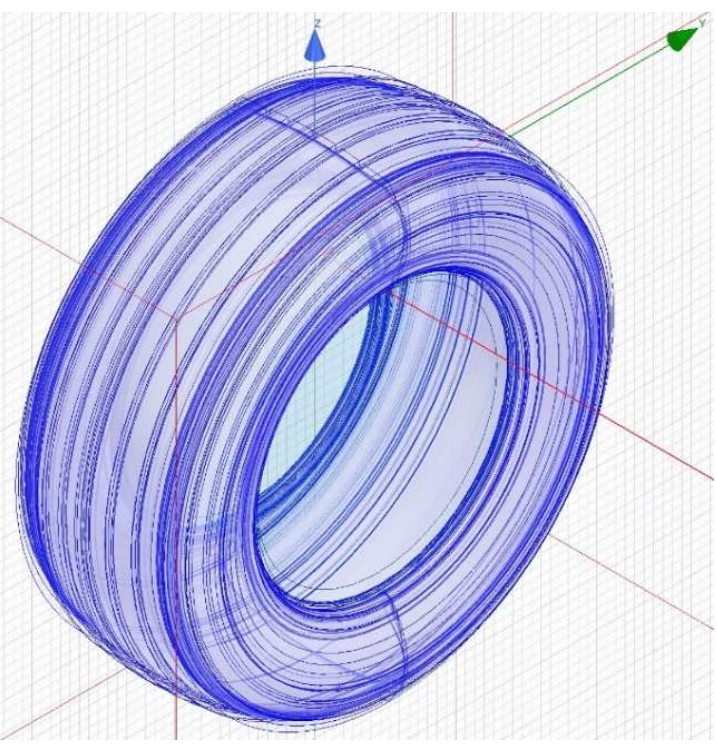

Figure 6: Detailed 3D tire model used in the analysis

\section{B. Analysis of RF propagation inside the tire and wheel}

In order to analyze RF propagation in the heavy vehicle environment, we place the transmitter model inside the tire on top of the middle section of the wheel barrel, along the circumference. The TPMS sensors are insulated and ruggedized by encasing them sensor in a plastic mold. The encasing was not modelled, but the transmitter was insulated from the wheel barrel by a layer of glass fiber reinforced plastic of relative permittivity 12 . This is the same material used in the TPMS incasing. The insulation reduces the attenuation effect of the steel barrel. Once the model is complete, we set the sweep setting, incident power and excitation parameters. Figure 7 and 8 present the propagation 


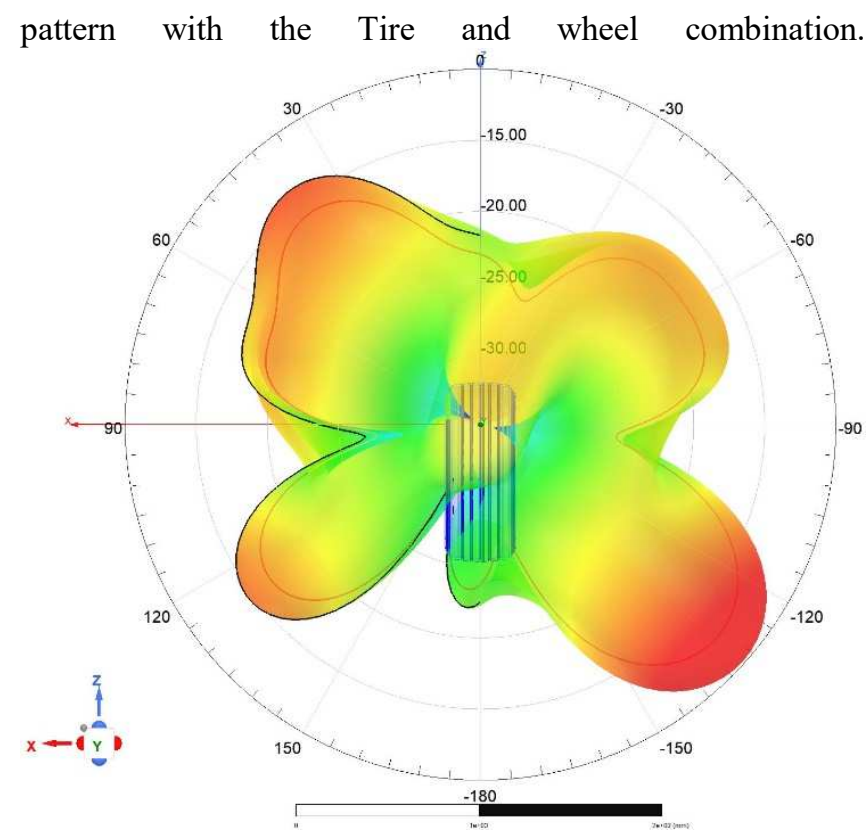

Figure 7: Propagation pattern along the $X$ and $Z$ axis with the Tire and Wheel

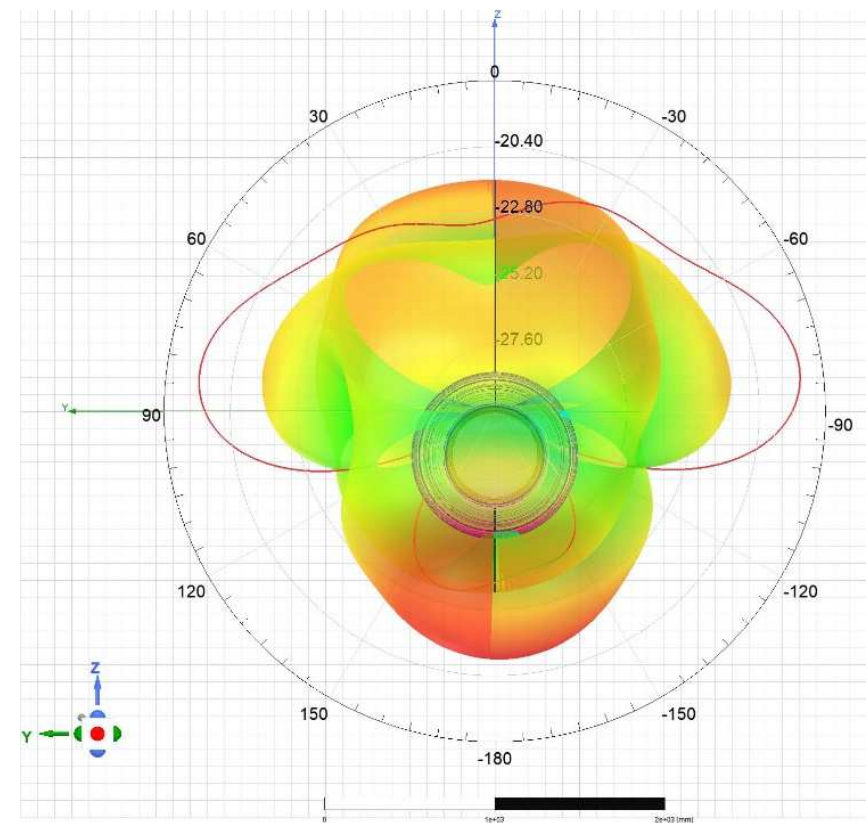

Figure 8: Propagation pattern along the $Y$ and $Z$ axis with the Tire and Wheel

At first, the signal propagation appears to have benefitted from the tire and wheel structure, with the maximum antenna gain increasing to $-12.6 \mathrm{~dB}$ but minimum gain is reduced to -34.9 $\mathrm{dB}$. This can be attributed to the steel wheel barrel extending the length electrically small antenna which would effectively increase the radiation efficiency. The wheel barrel also channels the radiation along the barrel. This has also given a degree of directivity along the X-Axis to the isotropic antenna propagation. Nevertheless, once the complete structure of the wheel and tire combination is taken into consideration, it can be seen that the radiation pattern is significantly distorted. The thick steel sheets of the wheel shields the RF signals completely causing null points in the propagation patterns, while the multiple layers of steel mesh in the tire form a barrier that greatly attenuate the signal. The RF signal propagates through the tire sidewalls and the tread area close to the transmitter. However, the signal suffers from multipath and is significantly weakened to an extent that the receiver unit needs to be close to the sensor to receive a signal. The analysis shows a maximum detection range of around 1.6 meters along the $\mathrm{Y}$ $\mathrm{Z}$ axis if the peak lobes propagating from the tire side walls are detected. Extensive infrastructure in both the tractor and trailer is required for the transmitters to be detected, which will increase costs and complexity. This makes interchangeability of trailers not possible.

As mentioned previously, placing the antenna inside the wheel and tire combination caused the antenna to detune. The operating frequency shifted to between $420 \mathrm{MHz}$ and $430 \mathrm{MHz}$ with a broad S-parameter $(\mathrm{S} 1,1)$ of $-34 \mathrm{~dB}$ to $-37 \mathrm{~dB}$ and VSMR of 0.07 , the analysis had to be carried out several times to determine the best tuning, a precise $434 \mathrm{MHz}$ operating frequency was not possible to achieve, but a range from $430 \mathrm{MHz}$ to $440 \mathrm{MHz}$ with an $\mathrm{S} 1,1$ of $-42 \mathrm{~dB}$. However, this is not a fixed value as changes to the wheel and tire specifications detune the antenna again. TPMS systems have a bandwidth of only several hundred Kilo-Herz to accommodate for several transmitters, the antenna tuning achieved was expected as this particular advanced antenna design has a broader bandwidth and a lower quality factor to help match the antenna to the surrounding environment [12]. On the other hand, this detuning indicates a detrimental effect of the surrounding environment on the antenna efficiency, which will increase once other factors affecting the antenna are taken into consideration. This may cause the antenna to be out of the tuning range and thus inefficient. Figures 9 and 10 show S-Parameter and Voltage Standing Wave Ratio (VSWR) of the antenna and the frequency shift due to the antenna detuning by the wheel and tire combination.

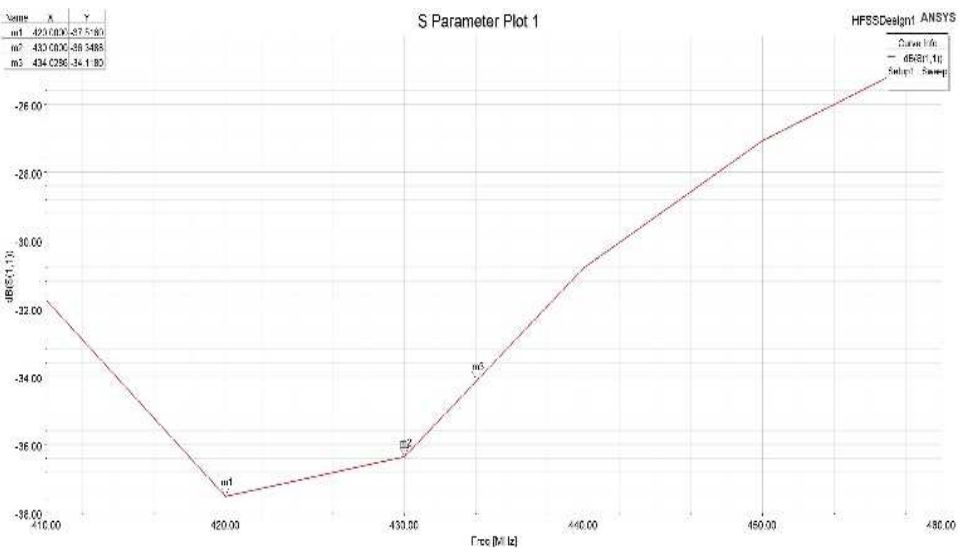

Figure 9: S-Parameter of the antenna inside the wheel and tire

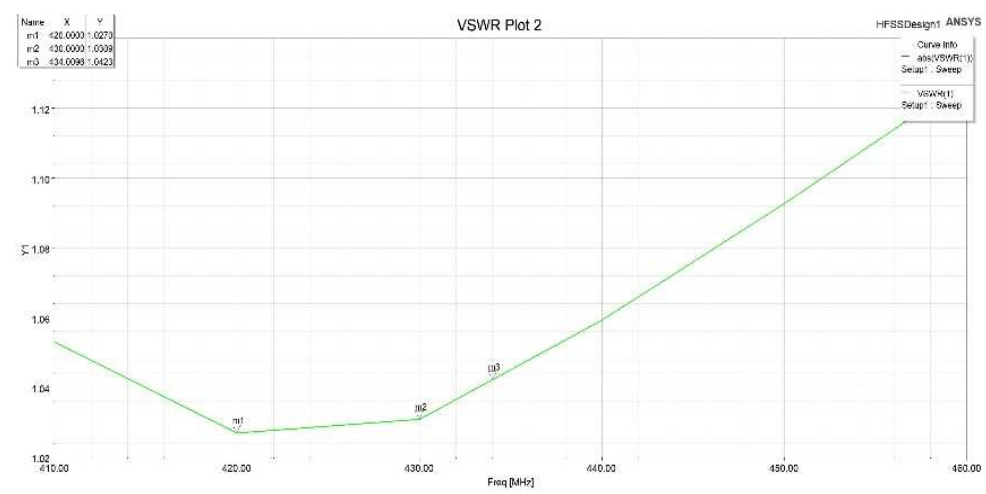

Figure 10: VSWR of the antenna inside the wheel and tire 


\section{OTHER FACTOR AFFECTING RF PROPAGATION}

In addition to the factors evaluated in Section III, there are other factors that affect RF signal propagation in the heavy vehicle environment: Weather conditions impedes the signal propagation in the carrier frequency used in the TPMS transmitter. Signal attenuation occurs in heavy rain and wet conditions are often encountered in long haul transportation in Europe; water will absorb the RF energy and prevent robust communication.

The tractor-trailer structure is also another important factor since the trailers consist of a long and thick steel beams, with several axles and wheel hubs together with spring leaf or coil leaf suspension and steel drum brakes [16][17]. All of which are made of thick gauge steel that attenuate the RF signal and detunes the transmission antenna. The tractor itself has a similar setup but at a less extent as shown in Figure 11 [17]. The tire and wheel combination is surrounded by these structures and the RF signal propagating through the tires sidewalls will need to propagate along all these structures to reach the reader unit, further attenuating the signal.

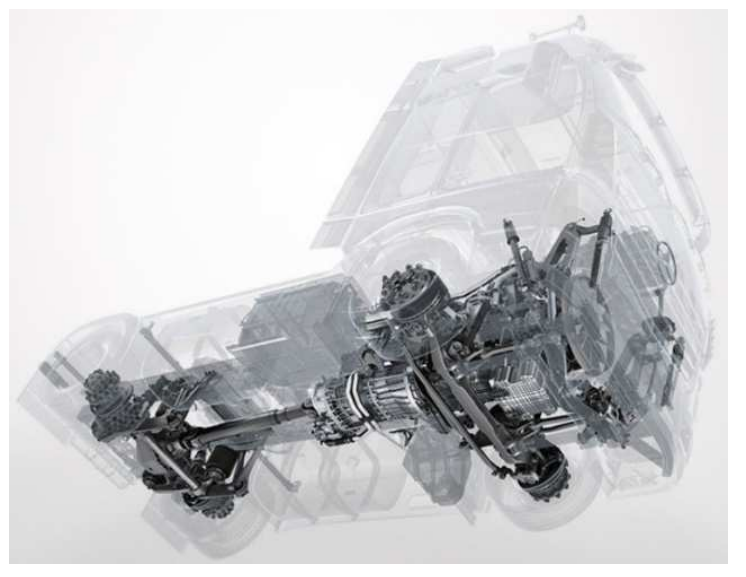

Figure 11: Internal structure of a heavy Tractor Truck [17]

We plan to evaluate the effect of the Tractor-Trailer structure on the RF signal propagation in an upcoming paper.

\section{CONCLUSION}

While industry has fully embraced the importance of TPMS, the majority of studies consider simplified models for finite element analysis, which would provide inaccurate output and measurements, which in turn leads to misleading conclusion. While many previous studies reported signal propagation evaluation between the transmitter and receiver of tire pressure information in passenger cars, few studies have considered heavy vehicles given their peculiarities. To fill this gap, this paper focuses on heavy commercial vehicles and provided an accurate modelling of the geometry, material, tire and wheel. We have demonstrated how truck structure and used materials impedes drastically signal propagation between the transmitter and receiver making the TPMS system communication method highly inefficient, and inoperable in some cases. This is confirmed by the Maximum power output settings currently being used in these systems, which lead to significantly reduced service life of the TPMS sensors in comparison to theoretical calculations. The antenna system is highly sensitive to detuning, and has to be matched to the application environment, and so changes to the wheel and tire type, shape or size may detune the antenna and cause detection errors. Thus, an alternative communication technology is needed for harsh environments with high density of metals and liquids. This will be the objective of our future research.

\section{ACKNOWLEDGMENT}

This work was undertaken under the NF Tires project 12513327, funded by the National Research Fund Luxembourg (FNR).

\section{REFERENCES}

[1] Goodyear Proactive Solutions, "Proactive Tire," 2021. [Online]. Available: https://www.proactive.goodyear.com/solution/goodyeartpms/. [Accessed: 2-Jan-2021]

[2] N. Q. Dinh, N. Michishita, Y. Yamada, and K. Nakatani, "Electrical characteristics of a very small normal mode helical antenna mounted on a wheel in the TPMS application," Proc. of IEEE International Symposium on Antennas and Propagation, Charleston, SC, June 2009, pp. 1-4.

[3] Continental Tires GmbH, "ContiPressurcheck," 2021. [Online]. Available:https://www.continentaltires.com/transport/products/overvi ew-product-lines/contipressurecheck/about. [Accessed: 2-Jan-2021]

[4] The Economic commission of European Union, "Regulation number 64 for Uniform provisions concerning the approval of vehicles with regard to their equipment which may include: a temporary-use spare unit, run-flat tyres and/or a run-flat system, and/or a tyre pressure monitoring system," 2010. [Online]. Available: https://op.europa.eu/en/publication-detail/-/publication/cce1a9e937b7-4973-bb70-d64999ba110c/language-en. [Accessed: 2-Jan-2021]

[5] D.Pozar. Microwave Engineering, third edition, John Wiley \& Sons, 2004

[6] A. Özdemirli, M. M. Bilgic and K. Yegin, "TPMS receive antenna design for large vehicles," 2011 XXXth URSI General Assembly and Scientific Symposium, Istanbul, 2011.

[7] H. Zeng and T. Hubing, "The effect of the vehicle body on EM propagation in tire pressure monitoring systems," IEEE Trans. on Antennas and Propagation, vol. 60, no. 8, Aug. 2012, pp. 3941-3949

[8] Dinh, N. Q. et al. "FEKO-based method for electromagnetic simulation of carcass wires embedded in vehicle tires." Applied Computational Electromagnetics Society Journal 26 (2011): 217-224.

[9] T. Teranishi, N. Dinh, N. Michishita, Y. Yamada and K. Nakatani, "Simulations of Radiation from an Antenna Contained in a Tire Equipped with Carcass", Proc. International Symposium on Antennas and Propagation (ISAP), 2009

[10] Cheikh, Mohamed et al. "RF source characterization of Tire Pressure Monitoring System." 2009 European Wireless Technology Conference (2009): 176-179

[11] Ansys.Inc, "HFSS: 3D Electromagnetic Field Simulator for RF and Wireless Design," 2021. [Online]. Available: https://www.ansys.com/products/electronics/ansys-hfss. [Accessed: 2Jan-2021

[12] Zeng, Hua and T. Hubing. "Investigation of Antennas used in Tire Pressure Monitoring Systems." (2010) technical report: CVEL-10-023

[13] H. Zeng and T. Hubing, "Tire Pressure Monitoring System (TPMS) EM Propagation Modeling Progress Part 1: Antenna Design," Clemson Vehicular Electronics Laboratory Technical Report, CVEL-09-009, Oct. 5, 2009

[14] U.S. Tire manufacturers association "Care and service of commercial truck and bus tires" Maintenance manual [accessed: 2-Jan-2021]

[15] J. Grosinger, L. W. Mayer, C. F. Mecklenbrauker and A. L. Scholtz, "Determining the Dielectric Properties of a Car Tire for an Advanced Tire Monitoring System," 2009 IEEE 70th Vehicular Technology Conference Fall, Anchorage, AK, 2009, pp. 1-5.

[16] Mercedes-Benz Trucks "ACTROS-Specifications" Specification manual, number 0001546002 [accessed: 2-Jan-2021]

[17] Mercedes-Benz Trucks "Actros: Frame, chassis, suspension, steering" 2021 [Online]. Available: https://www.mercedes-benz-trucks.com. [Accessed: 2-Jan-2020] 